\title{
Detection of outliers in multivariate data: a method based on clustering and robust estimators
}

\author{
Carla M. Santos-Pereira ${ }^{1}$ and Ana M. Pires ${ }^{2}$ \\ 1 Universidade Portucalense Infante D. Henrique, Oporto, Portugal and \\ Applied Mathematics Centre, IST, Technical University of Lisbon, \\ Portugal. \\ 2 Department of Mathematics and Applied Mathematics Centre, IST, \\ Technical University of Lisbon, Portugal.
}

Keywords. Multivariate analysis, Outlier detection, Robust estimation, Clustering, Supervised classification

\section{Introduction}

Outlier identification is important in many applications of multivariate analysis. Either because there is some specific interest in finding anomalous observations or as a pre-processing task before the application of some multivariate method, in order to preserve the results from possible harmful effects of those observations. It is also of great interest in supervised classification (or discriminant analysis) if, when predicting group membership, one wants to have the possibility of labelling an observation as "does not belong to any of the available groups". The identification of outliers in multivariate data is usually based on Mahalanobis distance. The use of robust estimates of the mean and the covariance matrix is advised in order to avoid the masking effect (Rousseeuw and Leroy, 1985; Rousseeuw and von Zomeren, 1990; Rocke and Woodruff, 1996; Becker and Gather, 1999). However, the performance of these rules is still highly dependent of multivariate normality of the bulk of the data. The aim of the method here described is to remove this dependence.

\section{Description of the method}

Consider a multivariate data set with $n$ observations in $p$ variables. The basic ideas of the method can be described in four steps:

1. Segment the $n$ points cloud (of perhaps complicated shape) in $k$ smaller subclouds using a partitioning clustering method with the hope that each subcloud (cluster) looks "more normal" than the original cloud.

2. Then apply a simultaneous multivariate outlier detection rule to each cluster by computing Mahalanobis-type distances from all the observations to all the clusters. An observation is considered an outlier if it is an outlier for every cluster. All the observations in a cluster may also be considered outliers if the relative size of that cluster is small (our proposal is less than $2 p+2$, since for smaller number of observations the covariance matrix estimates are very unreliable).

3 . Remove the observations detected in 2 and repeat 1 and 2 until no more observations are detected.

4. The final decision on whether all the observations belonging to a given cluster (not previously removed, that is with size greater than $2 p+1$ ) are outliers is based on a table of between clusters Mahalanobis-type distances. 
There is no need to fix $k$ in advance, we suggest to apply several values of $k$ and observe the results (there is of course a limit on $k$, which depends on the number of observations and the number of variables).

The choice of the partitioning clustering method is crucial and may depend on some previous exploratory analysis of the data: if a not too complicated shape is expected than the classical $k$-means method (which tends to produce hyperspherical clusters) is adequate; with more complicated shapes, methods more robust to the "spherical cluster" assumption (like for instance the model based clustering of Banfield and Raftery, 1992) are to be preferred. It is also necessary to take into account the size of the data set. For large data sets the method clara of Kaufman and Rousseeuw (1990) may be appropriate.

By a simultaneous multivariate outlier detection rule we mean a rule such that, for a multivariate normal sample of size $n_{j}$, no observation is identified as an outlier with probability $1-\alpha_{j}, j=1, \ldots, k$ (see Davies and Gather, 1993). If $\alpha_{j}=1-(1-\alpha)^{1 / k}$ an overall level $\alpha$ can be guaranteed for mixtures of $k$ multivariate normal distributions. An observation, $\mathbf{x}$, with squared Mahalanobis distance $d^{2}=\left(\mathbf{x}-\hat{\boldsymbol{\mu}}_{j}\right)^{T} \hat{\boldsymbol{\Sigma}}_{j}^{-1}\left(\mathbf{x}-\hat{\boldsymbol{\mu}}_{j}\right)$ greater than a detection limit $c\left(p, n_{j}, \alpha_{j}\right)$ is declared an outlier relatively to the $j$ th cloud. The constant $c\left(p, n_{j}, \alpha_{j}\right)$ is asymptotically $\chi_{p ; \beta}^{2}$, with $\beta=\left(1-\alpha_{j}\right)^{1 / n_{j}}$. However, as discussed in Becker and Gather (2001), for not very large samples and depending on the estimators $\hat{\boldsymbol{\mu}}_{j}$ and $\hat{\boldsymbol{\Sigma}}_{j}$, there may be large differences to the asymptotic value. Those authors suggest that more reliable constants can easily be determined by simulation. For the estimators $\hat{\boldsymbol{\mu}}_{j}$ and $\hat{\boldsymbol{\Sigma}}_{j}$ we may choose the classical sample mean vector and sample covariance matrix, or, for greater protection against masking, robust analogues of them.

In step 4 , let the observations (not removed in the previous steps) be labelled as $\mathbf{x}_{i j}, i=1, \ldots, n_{j}, j=1, \ldots, k$, where $n_{j}$ are the sizes of the final $k$ clusters (note that $\sum n_{j}$ may be smaller than $n$ ). Then define

$$
D_{l m}=\min _{i=1, \ldots, n_{l}}\left(\mathbf{x}_{i l}-\hat{\boldsymbol{\mu}}_{m}\right)^{T} \hat{\boldsymbol{\Sigma}}_{m}^{-1}\left(\mathbf{x}_{i l}-\hat{\boldsymbol{\mu}}_{m}\right) .
$$

A given cluster, $l$, is said to be disconnected from the remaining data if $D_{l m}>c\left(p, n_{m}, \alpha_{m}\right)$, for all $m \neq l$, and connected otherwise. Disconnected clusters are suspicious of containing only outliers, however such a decision is application dependent and must be taken case by case.

The proposed procedure is affine invariant (that is, the observations identified as outliers do not change under affine transformations) if the location and scatter estimators are affine equivariant and if the clustering method is affine invariant. Note that the $k$-means method does not have this property.

\section{Simulation study}

In order to evaluate the performance of the above method and to compare it with the usual method of a single Mahalanobis distance we conducted a simulation study with:

- Three clustering methods: $k$-means, pam (partioning around medoids, from Kaufman and Rousseeuw, 1990) and mclust (model based clustering for gaussian distributions, from Banfield and Raftery, 1992), each of them with $k=3,4,5$. 
- Two pairs of location-scatter estimators: classical $(\overline{\mathbf{x}}, \mathbf{S})$ and Reweighted Minimum Covariance Determinant (Rousseeuw, 1985) with an approximate $25 \%$ breakdown point (denoted RMCD25), which has better efficiency than the one with (maximal) \%50 breakdown point. (For the first estimators we used the asymptotic detection limits while for the RMCD25 the detection limits were determined previously by simulation with 10000 normal data sets.)

- Overall significance level: $\alpha=0.1$.

- Classify disconnected clusters as outliers.

- Eight distributional situations:

1. Normal $(p=2)$ without outliers, 150 observations from $N_{2}(\mathbf{0}, \mathbf{I})$.

2. Normal $(p=2)$ with outliers, 150 observations from $N_{2}(\mathbf{0}, \mathbf{I})$ plus 10 outlying observations from $N_{2}(\mathbf{1 0 . 5 1}, 0.1 \mathrm{I})$.

3. Normal $(p=4)$ without outliers, 150 observations from $N_{4}(\mathbf{0}, \mathbf{I})$.

4. Normal $(p=4)$ with outliers, 150 observations from $N_{4}(\mathbf{0}, \mathbf{I})$ plus 10 outlying observations from $N_{4}(\mathbf{8 . 5 9}, 0.1 \mathrm{I})$.

5. Non-normal $(p=2)$ without outliers, 50 observations from $N_{2}\left(\boldsymbol{\mu}_{1}, \boldsymbol{\Sigma}_{1}\right)$, 50 observations from $N_{2}\left(\boldsymbol{\mu}_{2}, \boldsymbol{\Sigma}_{2}\right)$ and 50 observations from $N_{2}\left(\mathbf{0}, \boldsymbol{\Sigma}_{1}\right)$, with $\boldsymbol{\mu}_{1}=(0,12)^{T}, \boldsymbol{\Sigma}_{1}=\operatorname{diag}(1,0.3), \boldsymbol{\mu}_{2}=(1.5,6)^{T}$ and $\boldsymbol{\Sigma}_{2}=$ $\operatorname{diag}(0.2,9)$.

6. Non-normal ( $p=2$ ) with outliers, 150 observations as in the previous case plus 10 outlying observations from $N_{2}\left((-2,6)^{T}, 0.01 \mathbf{I}\right)$

7. Non-normal $(p=4)$ without outliers, 50 observations from $N_{4}\left(\boldsymbol{\mu}_{3}, \boldsymbol{\Sigma}_{3}\right)$ 50 observations from $N_{4}\left(\boldsymbol{\mu}_{4}, \boldsymbol{\Sigma}_{4}\right)$ and 50 observations from $N_{4}\left(\mathbf{0}, \boldsymbol{\Sigma}_{3}\right)$, with $\boldsymbol{\mu}_{3}=(0,12,0,0)^{T}, \boldsymbol{\Sigma}_{3}=\operatorname{diag}(1,0.3,1,1), \boldsymbol{\mu}_{4}=(1.5,6,0,0)^{T}$ and $\boldsymbol{\Sigma}_{4}=\operatorname{diag}(0.2,9,1,1)$.

8. Non-normal $(p=4)$ with outliers, 150 observations as in the previous case plus 10 outlying observations from $N_{4}\left((-2,6,0,0)^{T}, 0.01 \mathbf{I}\right)$.

For each combination we run 100 simulations using the statistical package S-plus. Figure 1 shows one of the generated data sets for situation 6 . The superimposed detection contours for two of the methods show that the outlier detection with previous clustering is much more appropriate to this situation.
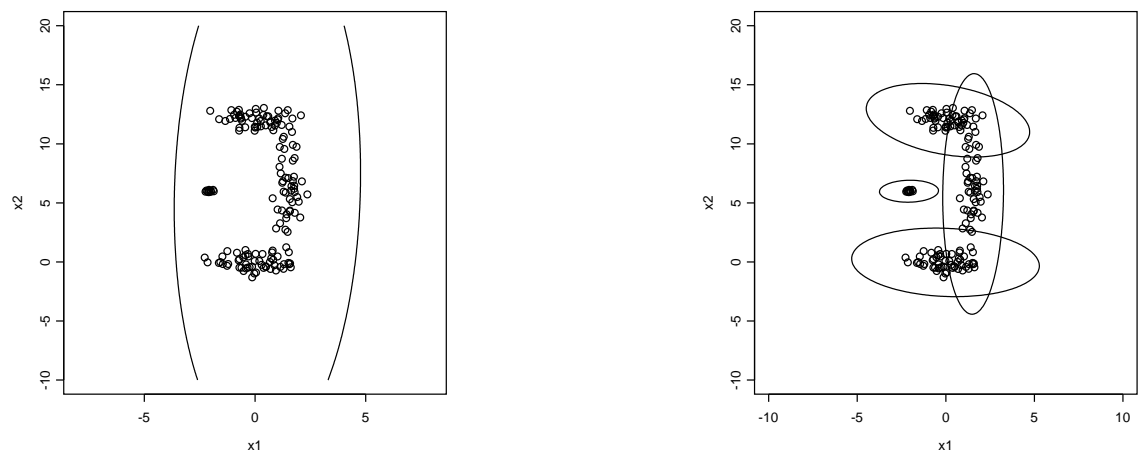

Fig. 1. Non-normal bivariate data with outliers and simultaneous detection contours $(\alpha=0.1)$ with: single robust Mahalanobis distance using RMCD25 (left panel); mclust with $k=4$ and RMCD25 (right panel). 
Tables 1 and 2 give the results of the simulation in terms of "proportion of runs with correct identification of all the outliers," $p_{1}$, "average proportion of outliers masked over the runs," $p_{2}$, "average proportion of swamping over the runs," $p_{3}$, and "proportion of runs without swamping" (see the simulation study of Kosinski, 1998). ${ }^{1}$ A good method should have $p_{1}=1, p_{2}=0$, small $p_{3}$ and $p_{4}=1-\alpha=0.9$. For the normal data all the methods behave very well, except for some masking with the classical Mahalanobis distance, which is not surprising. For nonnormal data the best performance is achieved by mclust with $k=4$ and $k=5$, without significant differences between the classical and the robust estimators. The failure of the $k$-means method is justified by the remarkable non-sphericity of the distributions chosen.

\section{Examples}

We have used the proposed method (with the same variants chosen for the simulation study) on the HBK data set (also analysed by several authors, like Rousseeuw and Leroy, 1985, or Rocke and Woodruff, 1996). All the variants detected exactly the 14 known outliers.

A more complicated data set was also analysed: the "pen-based automatic character recognition data" (available from http://www.ics.uci.edu/ mlearn /MLSummary.html). We have selected one of the classes, corresponding to the digit " 0 " with 1143 cases on 16 variables. An interesting feature of this data is that the characters can be plotted making it easy to judge the adequacy of the outlier detection (which is very convenient for checking on competing methods but very tedious to do for all the observations, besides the aim is to perform automatic classification). The single Mahalanobis distance with classical estimators revealed 106 outliers. The single Mahalanobis distance with RMCD25 pointed 513 observations (!!!) most of them looking quite unsuspicious. For the other options with clustering (only $k$-means and pam, since mclust is not adequate for data of this size) those numbers varied from 95 to 140 . Very aberrant observations were detected by all the methods including classical Mahalanobis distance. However, ten strange observations (looking more like a "6" than a " 0 ") were detected by all the clustering methods but not by the classical Mahalanobis distance. In future work we intend to assess the impact of this on the accuracy of the discrimination process for the whole data set.

\section{Conclusions}

From the simulation study and from the examples we conclude that the proposed method is promising. However, some refinements may be necessary. We intend to try other (more efficient) robust estimators and to perform a more extensive simulation study, namely to investigate the performance on larger data sets, higher contamination, and the instability of the indicator $p_{4}$.

\section{References}

Banfield, J.D. and Raftery, A.E. (1992). Model-based Gaussian and nonGaussian clustering. Biometrics, 49, 803-822.

Becker, C. and Gather, U. (1999). The masking breakdown point of multivariate outlier identification rules. Journal of the American Statistical Association, 94, 947-955.

\footnotetext{
${ }^{1}$ For the cases without outliers $p_{1}$ and $p_{2}$ are not defined (nd).
} 


\begin{tabular}{|c|c|c|c|c|c|c|c|c|c|c|}
\hline \multirow{2}{*}{ Situation } & \multirow{2}{*}{$\begin{array}{l}\text { Clust. } \\
\text { Method }\end{array}$} & \multicolumn{5}{|c|}{ MD with RMCD25 } & \multicolumn{4}{|c|}{ MD with $\overline{\mathbf{x}}$ and $\mathbf{S}$} \\
\hline & & $k$ & $p_{1}$ & $p_{2}$ & $p_{3}$ & $p_{4}$ & $p_{1}$ & $p_{2}$ & $p_{3}$ & $p_{4}$ \\
\hline \multirow{10}{*}{$\begin{array}{c}\text { Normal } \\
p=2 \\
\text { Without } \\
\text { outliers }\end{array}$} & $k$-means & 3 & nd & nd & 0.000 & 1.00 & nd & nd & 0.002 & 0.78 \\
\hline & & 4 & nd & nd & & & nd & nd & 0.001 & 0.87 \\
\hline & “ & 5 & nd & nd & 0.003 & 0.67 & nd & nd & 0.002 & 0.85 \\
\hline & pam & 3 & nd & nd & 0.000 & 1.00 & nd & nd & 0.002 & 0.78 \\
\hline & & 4 & nd & nd & 0.005 & & nd & nd & 0.002 & \\
\hline & " & 5 & nd & nd & 0.004 & 0.65 & nd & nd & 0.001 & 0.81 \\
\hline & mclust & 3 & nd & nd & 0.000 & 1.00 & nd & nd & 0.001 & 0.93 \\
\hline & & 4 & nd & nd & 0.003 & & nd & ad & 0.000 & $0 \pi$ \\
\hline & “ & 5 & nd & nd & 0.002 & 0.78 & nd & nd & 0.001 & 0.92 \\
\hline & none & 1 & nd & nd & 0.001 & 0.92 & nd & nd & 0.001 & 0.91 \\
\hline \multirow{10}{*}{$\begin{array}{c}\text { Normal } \\
p=2 \\
\text { With } \\
\text { outliers }\end{array}$} & -means & 3 & 1.00 & 0.00 & 0.000 & 1.00 & .00 & 0.00 & 0.002 & 0.1 \\
\hline & & 4 & 1.00 & 0.00 & 0.006 & 0.51 & 1.00 & 0.00 & 0.002 & 0.69 \\
\hline & " & 5 & 1.00 & 0.00 & & 0.62 & 1.00 & 0.00 & 0.001 & 0.85 \\
\hline & pam & 3 & 1.00 & 0.00 & 0.000 & 1.00 & 1.00 & 0.00 & 0.002 & 0.13 \\
\hline & & 4 & 1.00 & 0.00 & & 0.54 & 1.00 & 0.00 & 0.002 & 0.75 \\
\hline & $"$ & 5 & 1.00 & 0.00 & & 0.66 & 1.00 & 0.00 & 0.002 & 0.75 \\
\hline & mclust & 3 & 1.00 & 0.00 & 0.0 & 1.00 & 1.00 & 0.00 & 0.001 & 0.80 \\
\hline & & 4 & 1.00 & & & 0.72 & & 0.00 & 0.0 & 0.82 \\
\hline & $"$ & 5 & 1.00 & 0.00 & 0. & 0.72 & 1.00 & 0.00 & 0.001 & 0.9 \\
\hline & none & 1 & 1.00 & 0.00 & 0.001 & 0.88 & 0.84 & 0.16 & 0.001 & 0.93 \\
\hline \multirow{10}{*}{$\begin{array}{c}\text { Normal } \\
p=4 \\
\text { Without } \\
\text { outliers }\end{array}$} & $k$-means & 3 & nd & nd & 0.000 & 1.00 & nd & nd & 0.001 & 0.91 \\
\hline & & 4 & nd & nd & 0.001 & 0.94 & nd & nd & 0.000 & 0.9 \\
\hline & " & 5 & nd & nd & 0.000 & 0.99 & nd & nd & 0.000 & 0.99 \\
\hline & pam & 3 & nd & nd & 0.0 & 1.00 & nd & nd & 01 & 91 \\
\hline & & 4 & nd & nd & 0.0 & 0.93 & nd & nd & 0.001 & 0.93 \\
\hline & " & 5 & nd & nd & 0.000 & 0.95 & nd & nd & 0.000 & 0.99 \\
\hline & mclust & 3 & nd & nd & 0.0 & 1.00 & nd & nd & 0.000 & 0.9 \\
\hline & & 4 & nd & nd & 0. & 0. & nd & nd & 0.000 & 1.00 \\
\hline & " & 5 & nd & nd & 0.0 & 0.94 & nd & nd & 0.001 & 0.98 \\
\hline & none & 1 & nd & nd & & 0.95 & nd & nd & 0.001 & 0.91 \\
\hline \multirow{10}{*}{$\begin{array}{c}\text { Normal } \\
p=4 \\
\text { With } \\
\text { outliers }\end{array}$} & $c$-mea & 3 & 1.00 & 0.00 & 0.000 & 1.00 & .00 & 0.00 & 0.003 & 0.67 \\
\hline & & 4 & 1.00 & 0.00 & 0.007 & 0.44 & 1.00 & 0.0 & 0.003 & 0.69 \\
\hline & “ & 5 & 1.0 & 0.0 & & 0.5 & & 0.0 & & 0.82 \\
\hline & pam & $3 \mid$ & 1.00 & 0.00 & 0.0 & 1.00 & 1.00 & 0.00 & 0.002 & 0.76 \\
\hline & & 4 & 1.00 & 0.0 & 0.0 & 0. & 1.0 & 0.00 & 0.002 & 0.81 \\
\hline & " & 5 & 1.00 & 0.00 & & 0.65 & 10 & 0.0 & 0.001 & 0.87 \\
\hline & mclust & 3 & 1.00 & 0.00 & 0.0 & 1.00 & 1.0 & 0.0 & 0.002 & 0.80 \\
\hline & & 4 & 1.00 & 0.0 & & & 1.00 & 0.00 & 0.001 & 0.87 \\
\hline & 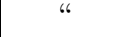 & 5 & 0.5 & 0.0 & & & & 0.0 & 0.001 & 0.89 \\
\hline & none & 1 & 1.00 & 0.0 & 0.0 & & .6 & 0.4 & 0.004 & 0.9 \\
\hline
\end{tabular}

Table 1. Results of the simulation study for normal data.

Becker, C. and Gather, U. (2001). The largest nonidentifiable outlier: a comparison of multivariate simultaneous outlier identification rules. Computational Statistics and Data Analysis, 36, 119-127.

Davies, P.L. and Gather, U. (1993). The identification of multiple outliers. Journal of the American Statistical Association, 88, 782-792.

Kaufman, L. and Rousseeuw, P.J. (1990). Finding Groups in Data: An Introduction to Cluster Analysis. New York: Wiley.

Kosinski, A.S. (1998). A procedure for the detection of multivariate outliers. Computational Statistics and Data Analysis, 29, 145-161.

Rocke, D.M. and Woodruff, D.L. (1996). Identification of outliers in multi- 


\begin{tabular}{|c|c|c|c|c|c|c|c|c|c|c|}
\hline \multirow[b]{2}{*}{ Situation } & \multirow{2}{*}{$\begin{array}{l}\text { Clust. } \\
\text { Method }\end{array}$} & \multirow[b]{2}{*}{$k$} & \multicolumn{4}{|c|}{ MD with RMCD25 } & \multicolumn{4}{|c|}{ MD with $\overline{\mathbf{x}}$ and $\mathbf{S}$} \\
\hline & & & $p_{1}$ & $p_{2}$ & $p_{3}$ & $p_{4}$ & $p_{1}$ & $p_{2}$ & $p_{3}$ & $p_{4}$ \\
\hline \multirow{10}{*}{$\begin{array}{c}\text { Non-normal } \\
p=2 \\
\text { Without } \\
\text { outliers }\end{array}$} & $k$-means & 3 & nd & nd & 0.199 & 0.14 & nd & nd & 0.063 & 0.60 \\
\hline & " & 4 & nd & nd & 0.017 & 0.77 & nd & nd & 0.037 & 0.72 \\
\hline & "6 & 5 & nd & nd & 0.005 & 0.82 & nd & nd & 0.022 & 0.77 \\
\hline & pam & 3 & nd & nd & 0.193 & 0.16 & nd & nd & 0.029 & 0.74 \\
\hline & 6 & 4 & nd & nd & 0.012 & 0.73 & nd & nd & 0.030 & 0.63 \\
\hline & $" 6$ & 5 & nd & nd & 0.006 & 0.70 & nd & nd & 0.036 & 0.73 \\
\hline & mclust & 3 & nd & nd & 0.046 & 0.81 & nd & nd & 0.041 & 0.73 \\
\hline & " & 4 & nd & nd & 0.018 & 0.71 & nd & nd & 0.044 & 0.75 \\
\hline & " & 5 & nd & nd & 0.001 & 0.83 & nd & nd & 0.024 & 0.84 \\
\hline & none & 1 & nd & nd & 0.001 & 0.90 & nd & nd & 0.000 & 0.99 \\
\hline \multirow{10}{*}{$\begin{array}{c}\text { Non-normal } \\
p=2 \\
\text { With } \\
\text { outliers }\end{array}$} & $k$-means & 3 & 0.94 & 0.06 & 0.213 & 0.06 & 0.14 & 0.86 & 0.035 & 0.81 \\
\hline & $" 6$ & 4 & 0.38 & 0.62 & 0.104 & 0.52 & 0.00 & 1.00 & 0.067 & 0.61 \\
\hline & "6 & 5 & 0.50 & 0.50 & 0.023 & 0.75 & 0.51 & 0.49 & 0.040 & 0.72 \\
\hline & pam & 3 & 0.89 & 0.11 & 0.207 & 0.11 & 0.18 & 0.82 & 0.046 & 0.73 \\
\hline & " & 4 & 0.84 & 0.16 & 0.169 & 0.23 & 0.00 & 1.00 & 0.045 & 0.74 \\
\hline & 6 & 5 & 0.83 & 0.17 & 0.008 & 0.72 & 0.53 & 0.47 & 0.045 & 0.71 \\
\hline & mclust & 3 & 0.21 & 0.79 & 0.054 & 0.78 & 0.17 & 0.83 & 0.047 & 0.75 \\
\hline & 6 & 4 & 0.99 & 0.01 & 0.026 & 0.80 & 0.99 & 0.01 & 0.043 & 0.73 \\
\hline & " & 5 & 0.94 & 0.06 & 0.009 & 0.80 & 1.00 & 0.00 & 0.014 & 0.85 \\
\hline & none & 1 & 0.00 & 1.00 & 0.000 & 0.96 & 0.00 & 1.00 & 0.000 & 1.00 \\
\hline \multirow{10}{*}{$\begin{array}{c}\text { Non-normal } \\
p=4 \\
\text { Without } \\
\text { outliers }\end{array}$} & $k$-means & 3 & nd & nd & 0.181 & 0.25 & nd & nd & 0.018 & 0.78 \\
\hline & $" 6$ & 4 & nd & nd & 0.028 & 0.71 & nd & nd & 0.026 & 0.82 \\
\hline & $"$ & 5 & nd & nd & 0.008 & 0.85 & nd & nd & 0.040 & 0.76 \\
\hline & pam & 3 & nd & nd & 0.124 & 0.47 & nd & nd & 0.016 & 0.80 \\
\hline & 66 & 4 & nd & nd & 0.007 & 0.82 & nd & nd & 0.037 & 0.81 \\
\hline & $"$ & 5 & nd & nd & 0.005 & 0.89 & nd & nd & 0.033 & 0.76 \\
\hline & mclust & 3 & nd & nd & 0.034 & 0.86 & nd & nd & 0.006 & 0.90 \\
\hline & 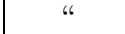 & 4 & nd & nd & 0.007 & 0.88 & nd & nd & 0.012 & 0.85 \\
\hline & 6 & 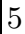 & nd & nd & 0.002 & 0.91 & nd & nd & 0.013 & 0.89 \\
\hline & none & 1 & nd & nd & 0.001 & 0.90 & nd & nd & 0.000 & 0.97 \\
\hline \multirow{10}{*}{$\begin{array}{c}\text { Non-normal } \\
p=4 \\
\text { With } \\
\text { outliers }\end{array}$} & $k$-means & 3 & 0.76 & 0.24 & 0.205 & 0.21 & 0.07 & 0.93 & 0.019 & 0.82 \\
\hline & 6 & 4 & 0.13 & 0.87 & 0.045 & 0.57 & 0.01 & 0.99 & 0.074 & 0.75 \\
\hline & " & 5 & 0.16 & 0.84 & 0.015 & 0.69 & 0.26 & 0.74 & 0.040 & 0.79 \\
\hline & pam & 3 & 0.66 & 0.34 & 0.168 & 0.33 & 0.06 & 0.94 & 0.016 & 0.79 \\
\hline & 66 & 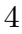 & 0.88 & 0.12 & 0.131 & 0.36 & 0.02 & 0.98 & 0.043 & 0.8 \\
\hline & $"$ & 5 & 0.74 & 0.26 & 0.013 & 0.79 & 0.21 & 0.79 & 0.042 & 0.78 \\
\hline & mclust & 3 & 0.23 & 0.77 & 0.291 & 0.53 & 0.18 & 0.82 & 0.171 & 0.64 \\
\hline & "6 & 4 & 1.00 & 0.00 & 0.013 & 0.84 & 0.99 & 0.01 & 0.009 & 0.88 \\
\hline & " & 5 & 0.94 & 0.06 & 0.007 & 0.92 & 0.98 & 0.02 & 0.015 & 0.90 \\
\hline & none & 1 & 0.00 & 1.00 & 0.001 & 0.90 & 0.00 & 1.00 & 0.000 & 0.96 \\
\hline
\end{tabular}

Table 2. Results of the simulation study for non-normal data.

variate data. Journal of the American Statistical Association, 91, 10471061.

Rousseeuw, P.J. (1985). Multivariate estimation with high breakdown point. In: Mathematical Statistics and Applications, Volume B, eds. W. Grossman, G. Pflug, I. Vincze and W. Werz 283-297. Dordrecht: Reidel.

Rousseeuw, P.J. and Leroy, A.M. (1985). Robust Regression and Outlier Detection. New York: Wiley.

Rousseeuw, P.J. and von Zomeren, B.C. (1990). Unmasking multivariate outliers and leverage points. Journal of the American Statistical Association, 85, 633-639. 